\title{
PARAMETRIC EQUATIONS FOR THE ENERGY GAP. EXTENDED BCS CASE
}

\author{
R. Gonczarek and M. Mulak \\ Institute of Physics, Technical University of Wrocław \\ Wybrzeże Wyspiańskiego 27, 50-370 Wrocław, Poland \\ (Received June 21, 1994; in final form September 1, 1994)
}

The generalised form of the gap equation which comprises also some non-BCS approaches is defined and solved. The parametric gap equations have been derived in a few equivalent cases and discussed in dissimilar limits. It is shown that the relations between the critical temperature and zero-temperature energy gap can be extended for all systems under consideration. The main benefits and incontestable advantage of the presented method are demonstrated with regard to the general BCS case.

PACS numbers: $74.20 .-\mathrm{z}$

\section{Introduction}

Recently, some new mechanisms of pairing interaction are included in BCS-type superconductors [1-4]. The inclusion of additional factors modifies BCS Hamiltonian [5] and hence quasi-particle energy which can give rise to reconstruction of a gap equation. Though one can expect such enriched structure of the energy gap to imply new results the applied calculation methods have to be transposed from BCS formalism.

The purpose of this paper is to present the systematic calculation methods which are just general enough to apply to derive and evaluate the energy gap in generalised BCS case. The obtained results can be directly applied to specified models [1-5]. We also demonstrate in detail the main benefits and indisputable advantage of our method with respect to the general BCS case when external magnetic field and superflow are included.

\section{General form of a gap equation solution}

We assume that for a wide class of $S$-paired quasi-particle Fermi systems (superconductors) the gap equation can be written in the following general form (cf. [6]):

$$
1=\lambda \int_{0}^{\omega_{\mathrm{D}}} \frac{\mathrm{d} \xi}{\sqrt{\xi^{2}+\Delta^{2}(T)}} F\left(\frac{\xi}{2 T}, \frac{\Delta(T)}{2 T}, \frac{a}{2 T}, \frac{b}{2 T}\right),
$$


where $\lambda=N(0) g$ is the dimensionless coupling parameter, and $a$ and $b$ denote parameters connected with external fields e.g. a magnetic field and a superflow. We assume only about the function $F\left(l_{1}, l_{2}, l_{3}, l_{4}\right)$ that its properties are analogous as for BCS case i.e. it is restricted and in limiting cases can be approximated according to relations

$$
F\left(0, l_{2}, l_{3}, l_{4}\right) \sim l_{1}
$$

if

$$
l_{1} \rightarrow 0 \text { and }\left|l_{3}+l_{4}\right|<\left|l_{2}\right|
$$

and

$$
F\left(l_{1}, l_{2}, l_{3}, l_{4}\right)=F_{\infty}
$$

if $l_{1}$ or $l_{2}$ tends to infinity. The latter property of the function $F$ is also correct for other arguments $l_{3}$ and $l_{4}$, however it can be left out of account because the arguments have to fulfil the condition $\left|l_{3}+l_{4}\right|<\left|l_{2}\right|$ which ensures the stability of superconducting phase.

In BCS case the function $F\left(l_{1}, l_{2}, l_{3}, l_{4}\right)$ is of the form

$$
\begin{aligned}
& F(\left.\frac{\xi}{2 T}, \frac{\Delta(T)}{2 T}, \frac{\mu_{\mathrm{B}} H}{2 T}, \frac{p_{\mathrm{F}} V_{\mathrm{s}}}{2 T}\right) \\
& \quad=\frac{1}{4} \sum_{i=0}^{1} \int_{-1}^{1} \mathrm{~d} z \tanh \frac{\sqrt{\xi^{2}+\Delta^{2}(T)}+(-1)^{i} \mu_{\mathrm{B}} H+z p_{\mathrm{F}} V_{\mathrm{s}}}{2 T},
\end{aligned}
$$

where $H$ denotes an external magnetic field and $V_{\mathrm{s}}$ is bare quasi-particle superflow. We emphasise that these quantities should be replaced by their effective fields if the Fermi liquid interaction is included [6]. Note moreover that for BCS case $F_{\infty}=1$ and $F$ is an even function of $H$ and $V_{s}$.

Let us present now the calculation method which allows us to evaluate and solve the gap Eq. (1) effectively. To facilitate calculations we introduce dimensionless quantities

$$
U=\frac{\xi}{T_{\mathrm{c}}}, \quad X=\frac{T}{T_{\mathrm{c}}}, \quad Y=\frac{\Delta(T)}{T_{\mathrm{c}}}, \quad A=\frac{a}{T_{\mathrm{c}}}, \quad B=\frac{b}{T_{\mathrm{c}}},
$$

where $T_{\mathrm{c}}$ is an arbitrary constant in energy (temperature) scale. Now, after integration by parts Eq. (1) reduces to the form

$$
\begin{aligned}
\frac{1}{\lambda}= & \ln \left(\frac{2 \omega_{\mathrm{D}}}{T_{c}}\right) F_{\infty}-\ln (Y) F\left(0, \frac{Y}{2 X}, \frac{A}{2 X}, \frac{B}{2 X}\right) \\
& -\int_{0}^{\infty} \mathrm{d} U \ln \left(U+\sqrt{U^{2}+Y^{2}}\right) \frac{\partial}{\partial U} F\left(\frac{U}{2 X}, \frac{Y}{2 X}, \frac{A}{2 X}, \frac{B}{2 X}\right),
\end{aligned}
$$

where because of quick convergence of the integrand we have right to expand the upper limit of the integral up to infinity. Note that we can rewrite (5) in polar coordinates according to relations

$$
X=R(\varphi) \cos \varphi, \quad Y=R(\varphi) \sin \varphi,
$$

which allows us to obtain a very convenient parametric form of the energy gap. Before we do it let us introduce dimensionless quantities

$$
u=\frac{U}{2 X}=\frac{\xi}{2 T}, \quad \tau=\frac{Y}{2 X}=\frac{\Delta(T)}{2 T}=\frac{1}{2} \tan \varphi,
$$




$$
\alpha=\frac{A}{2 X}=\frac{a}{2 T}, \quad \beta=\frac{B}{2 X}=\frac{b}{2 T} \text { and } \frac{X}{Y}=2 \tau,
$$

which will definitively simplify the problem under consideration. Then, after some algebra, Eq. (6) can be reduced to the form

$$
\begin{aligned}
\frac{1}{\lambda}= & \ln \left(\frac{2 \omega_{\mathrm{D}}}{T_{\mathrm{c}}}\right) F_{\infty}-\ln (Y) F_{\infty} \\
& -\int_{0}^{\infty} \mathrm{d} u\left[\ln \left(u+\sqrt{u^{2}+\tau^{2}}\right)-\ln \tau\right] \frac{\partial}{\partial u}\left[F(u, \tau, \alpha, \beta)-F_{\infty}\right],
\end{aligned}
$$

or equivalently to

$$
\begin{aligned}
\frac{1}{\lambda}= & \ln \left(\frac{2 \omega_{\mathrm{D}}}{T_{c}}\right) F_{\infty}-\ln (2 X) F_{\infty}-\ln (\tau) F(0, \tau, \alpha, \beta) \\
& -\int_{0}^{\infty} \mathrm{d} u \ln \left(u+\sqrt{u^{2}+\tau^{2}}\right) \frac{\partial}{\partial u} F(u, \tau, \alpha, \beta) .
\end{aligned}
$$

Equations (9)-(10) allow us to write down the parametric equations for energy gap versus temperature in reduced units i.e. we get from Eq. (9) or Eq. (10)

$$
Y=Y(\tau) \text { or } X=X(\tau),
$$

respectively and moreover we have

$$
Y(\tau)=2 \tau X(\tau) \text { or } X(\tau)=\frac{1}{2 \tau} Y(\tau)
$$

However, the above equations contain two constants i.e. $\lambda$ and $\omega_{D}$ which are inconvenient for detailed evaluation. Fortunately, we can eliminate them from Eqs. (9)-(10) when replacing by one from other critical quantities such as critical temperature or zero-temperature energy gap. Note that Eqs. (9)-(10) are written in a form which allows for easy study of one of the limiting cases, $T=0$ or $\Delta=0$. Therefore, putting $T=0\left(Y=\Delta(0) / T_{c}, \tau=\infty\right)$ in Eq. (9) and $\Delta=0(X=1$, $\tau=0$ and $T=T_{\mathrm{c}}$, hereafter $T_{\mathrm{c}}$ stands for a critical temperature) in Eq. (10) we get

$$
\frac{1}{\lambda}=\ln \left(\frac{2 \omega_{\mathrm{D}}}{\Delta(0)}\right) F_{\infty}
$$

and

$$
\frac{1}{\lambda}=\ln \frac{\omega_{\mathrm{D}}}{T_{\mathrm{c}}}-Q
$$

respectively where

$$
Q=\int_{0}^{\infty} \mathrm{d} u \ln (2 u) \frac{\partial}{\partial u} F(u, 0,0,0)
$$

must be a number in all possible cases and for BCS case $Q=\ln \left(\pi / 2 \mathrm{e}^{C}\right)$. Comparing Eqs. (12) and (13) we state that the obtained formula

$$
\Delta(0)=2 T_{\mathrm{c}} \exp \left(\frac{Q}{F_{\infty}}\right)
$$

is of BCS-type, hence $\Delta(0)$ must be proportional to $T_{\mathrm{c}}$ for all cases under discussion. Employing relations among $\lambda, \omega_{\mathrm{D}}, T_{\mathrm{c}}$ and $\Delta(0)$, as derived above, we can 
eliminate $\lambda$ and $\omega_{\mathrm{D}}$ from Eqs. (9)-(10) and get two equivalent sets of parametric gap equations which are of the form

$$
\begin{aligned}
& \ln (X) F_{\infty}=Q-\ln (\tau) F(0, \tau, \alpha, \beta) \\
& -\int_{0}^{\infty} \mathrm{d} u \ln \left(u+\sqrt{u^{2}+\tau^{2}}\right) \frac{\partial}{\partial u} F(u, \tau, \alpha, \beta)
\end{aligned}
$$

or

$$
\begin{aligned}
& \ln (Y) F_{\infty}=Q+\ln 2 \\
& \quad-\int_{0}^{\infty} \mathrm{d} u\left[\ln \left(u+\sqrt{u^{2}+\tau^{2}}\right)-\ln \tau\right] \frac{\partial}{\partial u} F(u, \tau, \alpha, \beta),
\end{aligned}
$$

where complementary quantities $X$ and $Y$ always can be found from relation $Y(\tau)=2 \tau X(\tau)$. The obtained integral representations of the energy gap are very convenient for numerical computing because of the strong convergence of integrands. However, in order to derive their analytical form which is available in the limit cases $T \rightarrow 0(\tau \rightarrow \infty)$ and $T \rightarrow T_{\mathrm{c}}(\tau \rightarrow 0)$, we have to transform them employing the following integral relations:

$$
\begin{gathered}
\int_{0}^{\infty} \mathrm{d} u\left[\ln \left(u+\sqrt{u^{2}+\tau^{2}}\right)-\ln \tau\right] \frac{\partial}{\partial u}\left[F(u, \tau, \alpha, \beta)-F_{\infty}\right] \\
=-\int_{0}^{\infty} \mathrm{d} u \frac{F(u, \tau, \alpha, \beta)-F_{\infty}}{\sqrt{u^{2}+\tau^{2}}}
\end{gathered}
$$

and

$$
\begin{gathered}
\int_{0}^{\infty} \mathrm{d} u\left[\ln \left(u+\sqrt{u^{2}+\tau^{2}}\right) \frac{\partial}{\partial u} F(u, \tau, \alpha, \beta)-\ln (2 u) \frac{\partial}{\partial u} F(u, 0,0,0)\right] \\
=\ln (\tau) F(0, \tau, \alpha, \beta)-\int_{0}^{\infty} \mathrm{d} u\left[\frac{F(u, \tau, \alpha, \beta)}{\sqrt{u^{2}+\tau^{2}}}-\frac{F(u, 0,0,0)}{u}\right] .
\end{gathered}
$$

Then Eqs. (15)-(16) reduce to the form

$$
\ln \frac{T}{T_{\mathrm{c}}}=\frac{1}{F_{\infty}} \int_{0}^{\infty} \mathrm{d} u\left[\frac{F(u, \tau, \alpha, \beta)}{\sqrt{u^{2}+\tau^{2}}}-\frac{F(u, 0,0,0)}{u}\right],
$$

and

$$
\ln \frac{\Delta(T)}{\Delta(0)}=\frac{1}{F_{\infty}} \int_{0}^{\infty} \mathrm{d} u \frac{F(u, \tau, \alpha, \beta)-F_{\infty}}{\sqrt{u^{2}+\tau^{2}}},
$$

where we also employed Eqs. (13) and (14).

\section{Application of the method for general BCS case}

Let us illustrate the developed method for the general BCS case when the function $F$ is defined by Eq. (4). 


\subsection{Numerical results}

In order to obtain the numerical results we transform Eq. (16) to the form

$$
\begin{aligned}
& Y(\tau)=\frac{\Delta}{T_{\mathrm{c}}}=\pi \mathrm{e}^{-C} \exp \left[-\frac{1}{2} \int_{0}^{\infty} \mathrm{d} u u \frac{\ln \left(u+\sqrt{u^{2}+\tau^{2}}\right)}{\sqrt{u^{2}+\tau^{2}}}\right. \\
& \left.\times \sum_{i=0}^{1} \frac{\tanh \left(\sqrt{u^{2}+\tau^{2}}+\chi+(-1)^{i} \omega\right)-\tanh \left(\sqrt{u^{2}+\tau^{2}}-\chi+(-1)^{i} \omega\right)}{2 \omega}\right] .
\end{aligned}
$$

Then

and

$$
X(\tau)=\frac{1}{2 \tau} Y(\tau)
$$

$$
R(\tau)=\frac{\sqrt{1+4 \tau^{2}}}{2 \tau} Y(\tau)
$$

is a slowly varying function of its arguments. The quantities $\chi$ and $\omega$ represent additional fields and are functions of $\tau$ as given by

$$
\chi=\frac{1}{2} \pi e^{-C} \frac{\sqrt{1+4 \tau^{2}}}{R(\tau)} h,
$$

and

$$
\omega=\frac{1}{2} \pi \mathrm{e}^{-C} \frac{\sqrt{1+4 \tau^{2}}}{R(\tau)} v
$$

where $h=\mu_{\mathrm{B}} H / \Delta(0)$ and $v=p_{\mathrm{F}} V_{\mathrm{s}} / \Delta(0)$ stand for reduced values of the magnetic field and superflow, respectively. Since $R(\tau)$ is a slowly varying function of $\tau$, in order to derive the shape of the energy gap for the fixed magnetic field and superflow we apply self-consistent iteration methods. The exemplary results obtained for a few chosen values of $h$ and $v$ are shown in Fig. 1.

\subsection{Analytical results}

Though there exist several equivalent methods of analytical research of the energy gap in two opposite limits i.e. $\tau=0$ and $\tau=\infty$, below we present a method elaborated by us which seems to be comprehensive and quick. The form of the energy gap in small $\tau$ limit $\left(T \rightarrow T_{\mathrm{c}}\right.$ ) can be found from Eq. (19) after series expansion of integrands according to $\tau, H$ and $V_{\mathrm{s}}$. Then after taking into account the following relations (cf. [6]):

$$
\begin{aligned}
& \int_{0}^{\infty} \frac{\mathrm{d} u}{u} \frac{\mathrm{d}}{\mathrm{d} u}\left(\frac{\tanh u}{u}\right)=-\frac{7 \zeta(3)}{\pi^{2}} \\
& \int_{0}^{\infty} \frac{\mathrm{d} u}{u} \frac{\mathrm{d}^{2} \tanh u}{\mathrm{~d} u^{2}}=-\frac{14 \zeta(3)}{\pi^{2}}
\end{aligned}
$$

Eq. (19) reduces to the form

$$
\ln \frac{T}{T_{\mathrm{c}}}=-\frac{7 \zeta(3)}{8 \pi^{2} T_{\mathrm{c}}^{2}}\left(\Delta^{2}+2 \mu_{\mathrm{B}}^{2} H^{2}+\frac{2}{3} p_{\mathrm{F}}^{2} V_{\mathrm{s}}^{2}\right)
$$



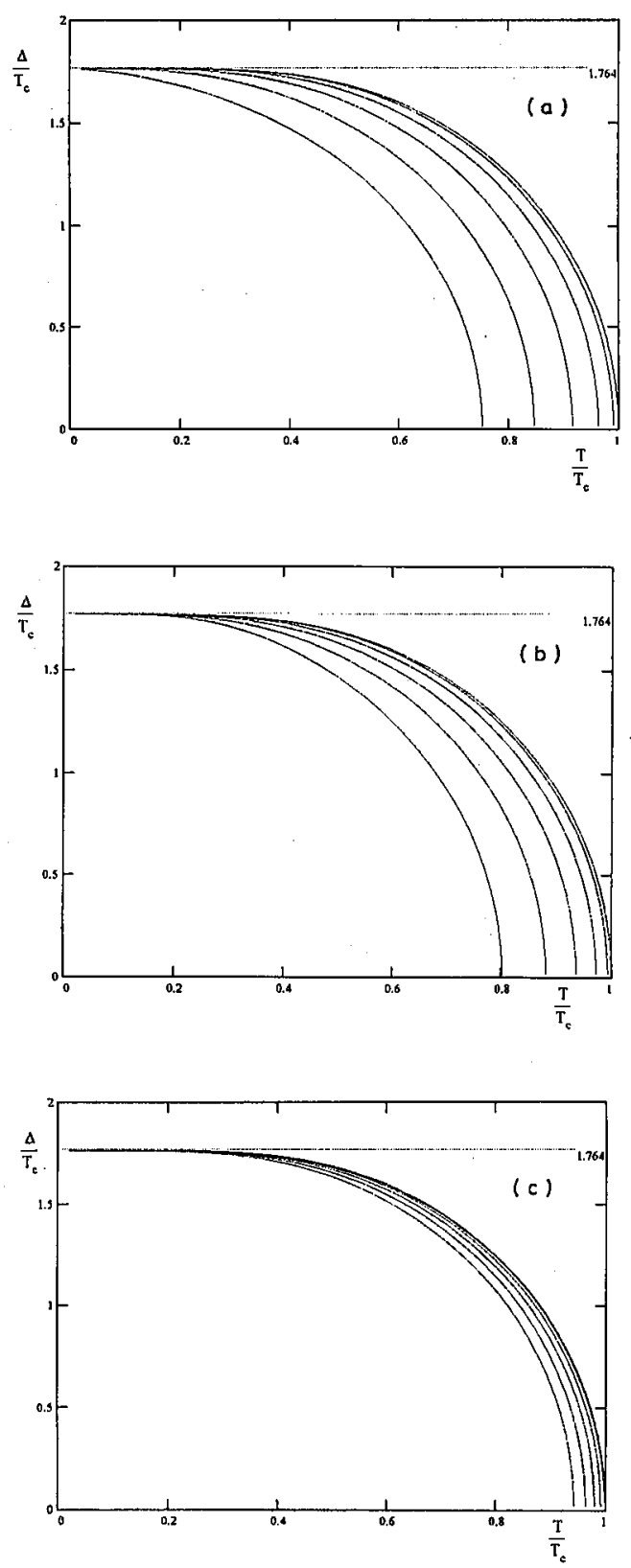

Fig. 1. The exemplary forms of the energy gap in dependence on reduced temperature for a few chosen values of $h$ and $v$. Note that the values of $\Delta$ lessen when values of $h$ and/or $v$ increase. (a) The succeeding curves obtained for the following values of $(h, v):(0,0),(0.1,0.1),(0.2,0.2),(0.3,0.3),(0.4,0.4),(0.5,0.5)$; (b) the succeeding curves obtained for the following values of $(h, v):(0,0),(0.1,0),(0.2,0),(0.3,0)$, $(0.4,0),(0.5,0)$; (c) the succeeding curves obtained for the following values of $(h, v)$ : $(0,0),(0,0.1),(0,0.2),(0,0.3),(0,0.4),(0,0.5)$. 
from which we obtain the following formula:

$$
\Delta=\left[\frac{8 \pi^{2} T_{\mathrm{c}}^{2}}{7 \zeta(3)}\left(1-\frac{T}{T_{\mathrm{c}}}\right)-2 \mu_{\mathrm{B}}^{2} H^{2}+\frac{2}{3} p_{\mathrm{F}}^{2} V_{\mathrm{s}}^{2}\right]^{\frac{1}{2}},
$$

where magnetic field and superflow must be sufficiently small quantities when compared to $\Delta$.

The case of large $\tau$ should be investigated with the aid of Eq. (20). Since in this case arguments of the function $F$ are always large we can replace hyperbolic tangent by exponential function according to the relation $\tanh \psi=1-2 \exp (-\psi)$, and then the integrands can be written down in the form

$$
-2 \exp \left(-2 \sqrt{u^{2}+\tau^{2}}-\ln \sqrt{u^{2}+\tau^{2}}\right) \cosh \frac{\mu_{\mathrm{B}} H}{T} \frac{\sinh \left(p_{\mathrm{F}} V_{\mathrm{s}} / T\right)}{p_{\mathrm{F}} V_{\mathrm{s}} / T}
$$

As the above integrand is strongly convergent at the point $u=0$, it can be replaced by the following expression:

$$
-2 \exp \left(-2 \tau-\ln \tau-\frac{1}{\tau} u^{2}\right) \cosh \left(\frac{\mu_{\mathrm{B}} H}{T}\right) \frac{\sinh \left(p_{\mathrm{F}} V_{\mathrm{s}} / T\right)}{p_{\mathrm{F}} V_{\mathrm{s}} / T} .
$$

Finally, after integration and simple algebra we obtain the following formula:

$$
\Delta=\Delta(0)-\sqrt{2 \pi T \Delta} \exp \left(-\frac{\Delta}{T}\right) \cosh \left(\frac{\mu_{\mathrm{B}} H}{T}\right) \frac{\sinh \left(p_{\mathrm{F}} V_{\mathrm{s}} / T\right)}{p_{\mathrm{F}} V_{\mathrm{s}} / T},
$$

which reduces to the well-known form [7] if we put $H$ and $V_{\mathrm{s}}$ equal to zero. In such a way the fundamental results of BCS theory for the energy gap have been repeated and generalized due to improvement and modification of analytical methods.

\section{Non-BCS case}

According to calculation given in [8] for the non-BCS system established in $[1,2]$ the structure of the gap equation coincides with Eq. (1) though the function $F$ becomes by far more complicated. However, employing the method presented above we can easily derive the relation between zero-temperature energy gap and critical temperature in accordance with Eq. (14). Appearing in Eq. (14), the function $Q$ is of the form

$$
Q=\int_{0}^{\infty} \mathrm{d} u \ln (2 u) \frac{\mathrm{d}}{\mathrm{d} u} \sum_{i=0}^{1} \frac{\sinh u}{2 \cosh u+4+3 \exp \left[(-1)^{i} u\right]} .
$$

Therefore, $Q=0.517$ and $F_{\infty}=\frac{5}{8}$. Hence, making use of Eq. (14) we find that for the discussed non-BCS case the following relation is fulfilled:

$$
\Delta(0)=2.287 T_{c}
$$

which ensures $\Delta(0)_{[\mathrm{BCS}]}<\Delta(0)$ on about 23 per cent.

\section{Conclusions}

The obtained results show that the pairing interaction parameter $\lambda$ and Debye frequency $\omega_{D}$ can always be eliminated from equations by means of the 
critical temperature $T_{\mathrm{c}}$ or zero-temperature energy gap $\Delta(0)$. Moreover, the obtained results i.e. the parametric equations for the energy gap are equivalent in both approaches. It causes that the relation $\Delta(0) / T_{c}$ must be constant and independent of other parameters of theory, also in non BCS cases, though the constant coefficient, which is a number, can be modified in some other approaches.

This research has been supported in part by grant 2 P302 12506 of the State Committee for Scientific Research (Republic of Poland).

\section{References}

[1] J. Spałek, W. Wójcik, Phys. Rev. B 37, 1532 (1988).

[2] J. Spałek, Phys. Rev. B 40, 5180 (1989).

[3] D. Belitz, Phys. Rev. B 35, 1636 (1987).

[4] K. Hallberg, C.A. Balseiro, Phys. Rev. B 43, 10289 (1991).

[5] J. Bardeen, L. Cooper, J.R. Schrieffer, Phys. Rev. 108, 1175 (1957).

[6] R. Gonczarek, Acta Phys. Pol. A 71, 59 (1987).

[7] A.A. Abrikosov, Fundamentals of the Theory of Metals, North-Holland, Amsterdam 1988.

[8] J. Czerwonko, in:-Proc. \& Int. Conf. on Materials and Mechanism of Superconductivity High-Temperature Superconductors, Grenoble (France) 1994. 